\title{
Aspects of high strain rate industrial forging of Inconel 718
}

\author{
A. Reshetov ${ }^{1 *}$, N. Stefani ${ }^{1}$, O. Bylya ${ }^{1}$, B. Krishnamurthy ${ }^{1}$, P. Blackwell $^{2}$ \\ ${ }^{1}$ Advanced Forming Research Centre, University of Strathclyde, 85 Inchinnan Drive, Inchinnan, Renfrewshire, PA4 9LJ, UK \\ ${ }^{2}$ Department of Design, Manufacture and Engineering Management, University of Strathclyde, 75 Montrose Street, Glasgow, G1 \\ $1 X J, U K$
}

Keywords: Inconel 718, High Strain Rate, Metal Flow, Microstructure Evolution, FEM

*Corresponding author: Aleksey Reshetov, e-mail: aleksey.reshetov@strath.ac.uk

\begin{abstract}
The major part of all material and microstructural data used for the modelling of nickel superalloy forgings are obtained from uniaxial laboratory tests with limited plastic strain and very simple thermo-mechanical history. At the same time, new challenges in near-net shape industrial forging require a high level of reliability of modelling prediction of metal flow, for predicting the risk of defects and microstructural transformation. A few recently conducted benchmarking studies have shown that despite the availability of various material models (including microstructural ones) embedded in commercial FE software, in many cases, the level of prediction remains unsatisfactory. This is especially true for Fast Industrial Forging Processes (like screw press or hammer forgings). This paper suggests a methodology for processing the results from industrial forgings for obtaining robust data for calibration, validation and improvement of material and microstructural models. This also can provide additional information on the material science behind the microstructural phenomena, which are problematic to capture and study using simple uniaxial tests.
\end{abstract}

\section{Introduction}

Similar to the parable of blind men and an elephant, engineers and researchers have studied metal forming to great depth using piecewise perspectives and great strides have been made in our understanding of specific aspects of forging. On the verge of a new era of industrial growth, we are now faced with new challenges that require a holistic perspective and a more unified approach.

Developed in the 1960s, Inconel 718 has become a crucial precipitation-hardened nickel superalloy [1]. Using this as the basis, a number of other more advanced and complicated alloys, classified as the 718-type class of alloys, have been developed $[2,3]$. The mechanical properties and microstructural phenomena of these alloys have been studied extensively under laboratory conditions, which typically involves uniaxial tests with limited strain and simple thermo-mechanical histories. Data from these uniaxial tests are used to validate classical forging models, specifically Finite-Element (FE) models.

However, these alloys were developed to be utilised under high-temperature and high-pressure conditions and highly corrosive environments such as those found in aircraft turbines and compressors. To meet the stringent needs of aerospace applications, these alloys are expected to be forged to Near Net-Shape with a tailored microstructure within the components. This involves Fast Industrial Forging Processes (FIFP) with a high strain-rate, strain and complex thermo-mechanical histories.

While traditionally, a process engineering based approach has been sufficient for assessing the process effectiveness in lower strain rate forging (e.g. hydraulic press forging), they fall short in the robust prediction of metal flow, risk of defects and microstructural transformation in FIFP. Benchmarking studies conducted by the authors recently clearly showed (see Fig. 1 below) that the traditional modelling approach fails to predict the material flow and microstructure in even the simplest case of FIFP for IN718. 
Experiment: $R X \%$ vol.

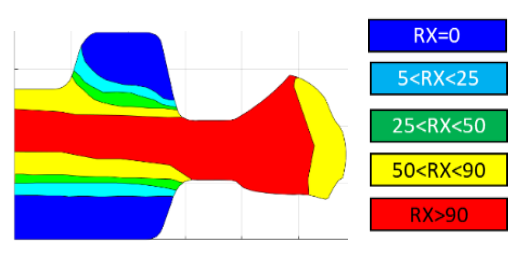

(a)

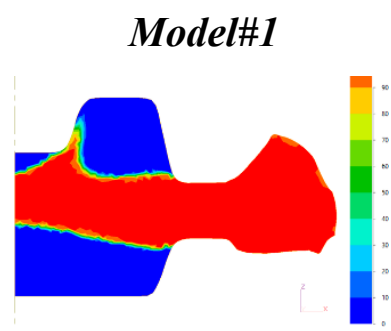

(b)

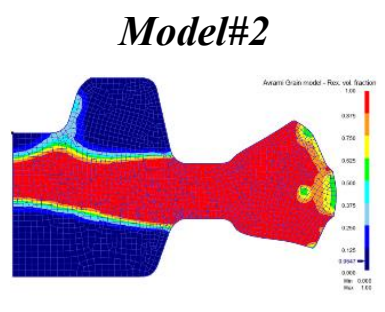

(c)
Model\#3

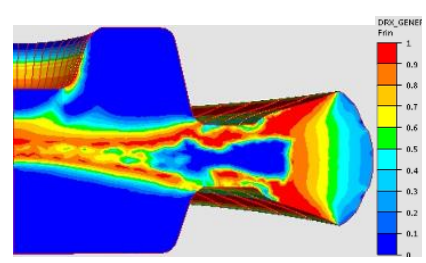

(d)

Figure 1. Results from benchmarking study of standard microstructure evolution models embedded into commercial FE software: (a) experimentally determined recrystallised volume fraction (RX\% vol.) in the cross-section of IN718 disk geometry forged with a screw press at $970^{\circ} \mathrm{C} ;(b),(c),(d)$ predictions of $\mathrm{RX} \%$ vol. by three different microstructure evolution models for IN718.

The main reason for the shortfall is linked to the fact that FIFP is a complex process not well represented by uniaxial tests. Therefore, this paper aims to analyse the problem of FIFP from different analytical viewpoints, namely, process engineering, solid mechanics and material science, which looks at the problem at different levels. The focus is laid on establishing a new methodology that will provide links between the different levels. Subsequently, using recent advances in data capture and finite-element modelling capabilities, an approach is suggested for robust calibration of material and microstructural models beyond the scope of uniaxial tests. Following this, successful case studies where such an approach can yield valuable results and insights are also provided.

\section{Approaches}

\section{1) Process engineering approach}

This is the predominantly adopted approach in manufacturing companies as it is the most practical approach possible in an industrial setting. Typically, this involves looking at a problem from the macro-level. A range of real-time processing parameters recorded on the forging press during forging such as position, velocity, force and energy as a function of time are used for the analysis. However, this data is not hugely informative when it comes to understanding the details of metal flow, or why specific defects or folds occur during the forging.

The knowledge gap is especially significant when it comes to FIFP, as these processes are characterised by a very high rate of loading. The velocity of tool impact on the workpiece can reach up to $1200 \mathrm{~mm} / \mathrm{s}$ in a screw press and it can go much higher, up to $9000 \mathrm{~mm} / \mathrm{s}$, in the case of hammers [4]. This, when coupled with a complicated geometry forged in single or multiple blows, results in complex thermal and strain histories [5]. It is not realistically possible to predict the resulting highly inhomogeneous deformation and complex microstructures using models derived from macro-level data. The solid mechanics approach described below sheds some useful light in this regard.

\section{2) Solid mechanics approach}

This approach uses continuum-based variables such as stress, strain and strain-rate to analyse a problem. FE modelling has become the preferred tool to perform this analysis lately. Phenomenological models are typically calibrated based on uniaxial tests that are embedded into FE packages to model the material flow. This approach faces two main limitations when it comes to modelling FIFP.

Firstly, the inability to account for the complicated history of loading in FIFP using lab tests. The tests are normally performed with constant strain-rate and temperature. The strain achievable in uniaxial tests is limited (to a max. $\varepsilon=0.7$ ) by the sample geometry, necking and frictional effects. Furthermore, it is non-trivial to perform high-strain tests in laboratories owing to machine limitations, complications in process control, and difficulties in processing the results. This results in short straight-line trajectories within narrow ranges of 
strain-rate and temperatures in the space of strain-strain-rate-temperature as shown in Fig. 2 below. In contrast, in FIFP, due to the different triaxiality factors, complex geometry, and multistage deformation, it is possible to reach very large plastic strains, easily an order higher than in uniaxial tests. Additionally, there exists a nonhomogenous distribution of temperature and strain-rate in a complex geometry FIFP. Therefore, the resulting trajectories are much longer and altogether of different shapes in FIFP than in uniaxial tests [6, 7], as can be observed in Fig. 2. This means that the major part of FE simulation of FIFP is performed using either interpolation or extrapolation of material data, which can lead to significant mistakes.

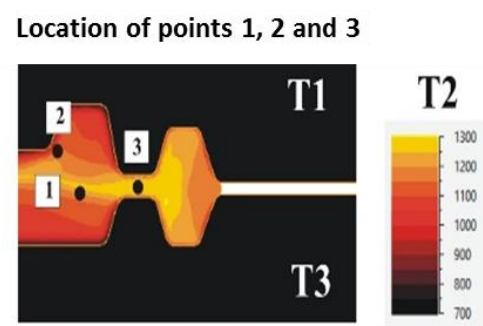

$\mathrm{T} 1 \neq \mathrm{T} 3-\mathrm{T} 1, \mathrm{~T} 3<<\mathrm{T} 2$
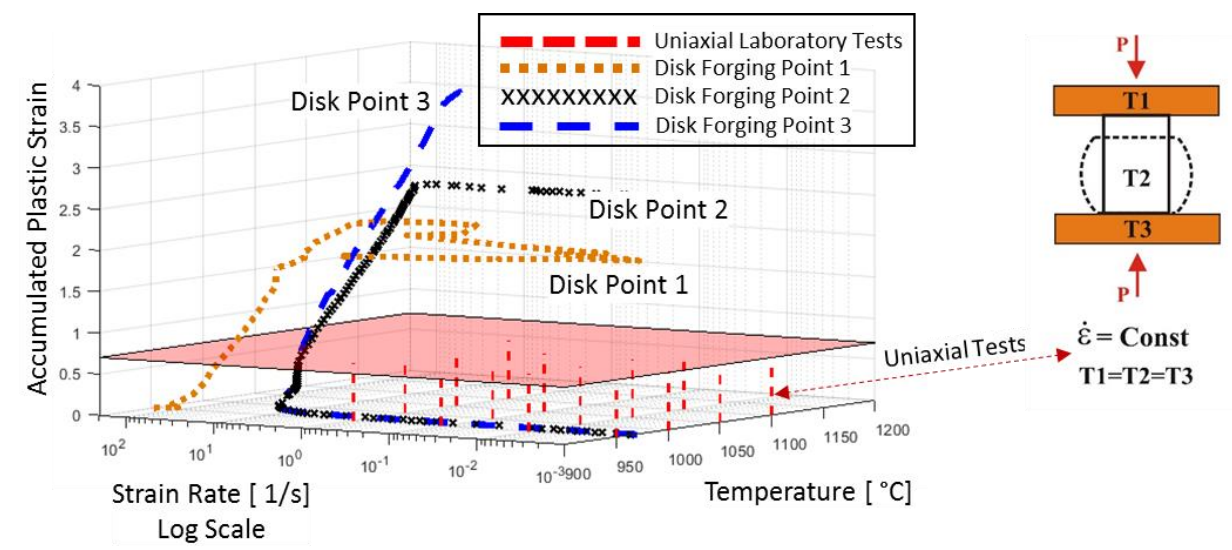

Log Scale

Figure 2. Comparison of thermo-mechanical history in reference points for uniaxial test and industrial-scale forging.

Secondly, FEA often fails to capture instabilities in metal flow in FIFP. The solid mechanics approach is not directly concerned with microstructural transformations, but uses phenomenological models to take them into account. Since they were developed mostly using simple processes (lab tests), the approximation falls short when the flow is complicated on account of multiple microstructural transformations. In FIFP, usually several microstructure evolution mechanisms act simultaneously. This is especially true in the case of IN718-type alloys. This can result in an unstable (transient) regime of metal flow. Moreover, these instabilities often occur at large strains, which again ties-in to the issues with extrapolation in FE models mentioned above. For instance, some materials exhibit drastic softening that leads to the formation of macroscopic shear bands, dead zones, etc. $[8,9]$. The most promising way to resolve this problem is to gain a better understanding of the nature of material behaviour at the microscopic level, which is possible from a material science approach described below.

\section{3) Material science approach}

Material science looks at material behaviour at a mesoscopic and microscopic level. Different microstructural mechanisms are often studied in isolation under specific conditions. Lab tests are carried out such that a single dominating microstructural mechanism occurs, and the resulting micro-sections are studied under optical or electron microscopes to formulate hypotheses. While they are hugely insightful, their applicability is limited to that particular mechanism and the conditions under which the studies were carried out.

FIFP covers a broad range of strain, strain rates and temperatures, and a mixture of microstructural evolution mechanisms can take place. Quite often, these mechanisms compete against each other. While some tend to stabilise metal flow, others might destabilise; while some cause softening, others lead to hardening. In addition, the elapsed time of the forging process is comparable to the time required for some of the microstructure evolution mechanisms to occur [10]. Therefore, some mechanisms are not activated/completed due to a lack of time, despite other conditions being met. To tackle this, further insights are needed to understand how microstructural transformation at high strain rates and strains differs from the well-studied deformation at low strains and velocities of loading [11, 12].

There have been attempts in the past to link these approaches and feed outputs from one level to the other. For instance, phenomenological models link microstructural transformation to solid mechanics. Multi-scale 
models are more advanced, and, as a result, more complex approaches for linking models of different levels. Processing maps $[13,14]$ were the first set of attempts to relate the material science approach to that of the process engineering approach. The limitations in using processing maps for FIFP are discussed in detail elsewhere [15]. Nevertheless, all these attempts involve models developed based on the very limited data obtained from uniaxial tests. On the other hand, there is plenty of data available from actual industrial forgings which are too complex or not readily intelligible to be used as data for calibration of material and microstructural models. Herein, a hybrid experimental-computational methodology is proposed to overcome this.

\section{Methodology}

\section{1) Forging and data capture}

The proposed methodology can be used for industrial forgings of different levels of complexity. In this paper, for illustration purposes, a double-truncated cone (DTC) and disk geometry shown in Fig. 3 were used.

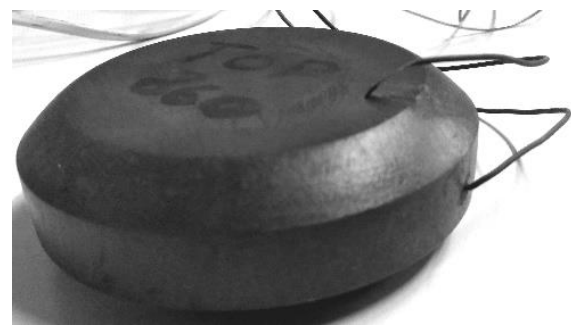

(a)

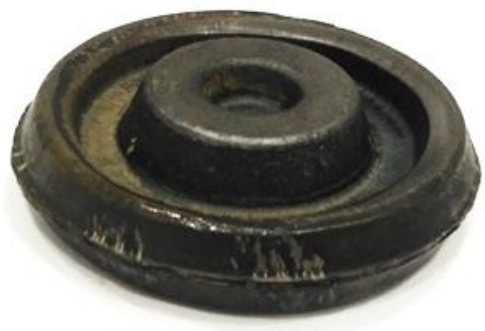

(b)

Figure 3. Representation of geometry used in the present study: (a) double-truncated cone; (b) profiled disk

Systematic industrial-scale forging trials were carried out on discs of IN718 using a Schuler AG 2100 tonne screw press with an upper ram velocity of $700 \mathrm{~mm} / \mathrm{s}$. The discs were forged at $970^{\circ} \mathrm{C}$ and $1080^{\circ} \mathrm{C}$. As per the process engineering approach, macro-level data such as position, velocity, force and energy as a function of time were recorded from the press for all trials. Furthermore, thermocouples carefully placed into the disc geometry were used for real-time temperature measurement. Preliminary FE simulations were carried out in the commercial FE package QForm to analyse the problem from a solid mechanics perspective. This was further extended to enable the link between the different approaches as described in the next section.

The forgings were sliced in half along the longitudinal axis for geometrical and microstructural evaluation. The forging microstructure was studied in three states: initial, half-forged and fully forged conditions. Data on the microstructure evolution under different forging conditions was collected using optical microscopy, scanning electron microscopy (SEM) and electron backscatter diffraction (EBSD) analysis. In addition, the entire cross-section of each forging was polished, etched and scanned on Alicona IFM G4 scanner to obtain macrographs and reveal metal flow patterns along the cross-section (see Fig. 4).

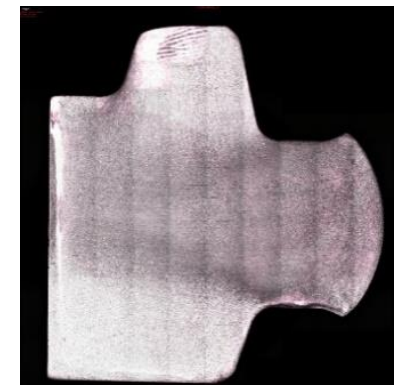

(a)

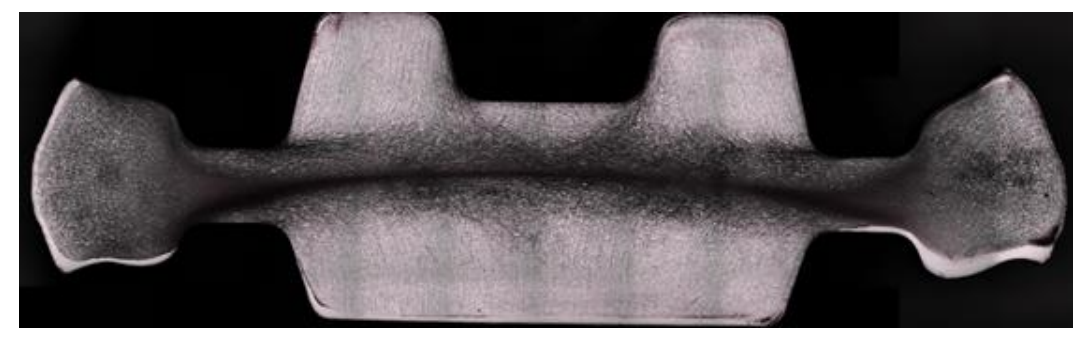

(b)

Figure 4. Macrographs of IN718 forged on a screw press at $970^{\circ} \mathrm{C}$ : (a) half-forged (one half of the crosssection) and (b) fully forged part. 


\section{2) Linkage of approaches}

At the core of the methodology suggested are "digital twins" of the forging process, which can link these seemingly different categories of data into a coherent set that can be used to understand the underlying mechanisms and thereby model the process. Digital twins are essentially representations of the actual forging process where all data are numerically quantified and are constructed as shown in Fig. 5 below. They solve two objectives, namely, (1) help digitise variables and parameters that are not directly measurable and (2) help to study the effect of one parameter in isolation while maintaining all others constant, which is not always possible in direct experiments. The key differences between digital twins and typical FE models are the overall objective of these tools and the level of accuracy involved.

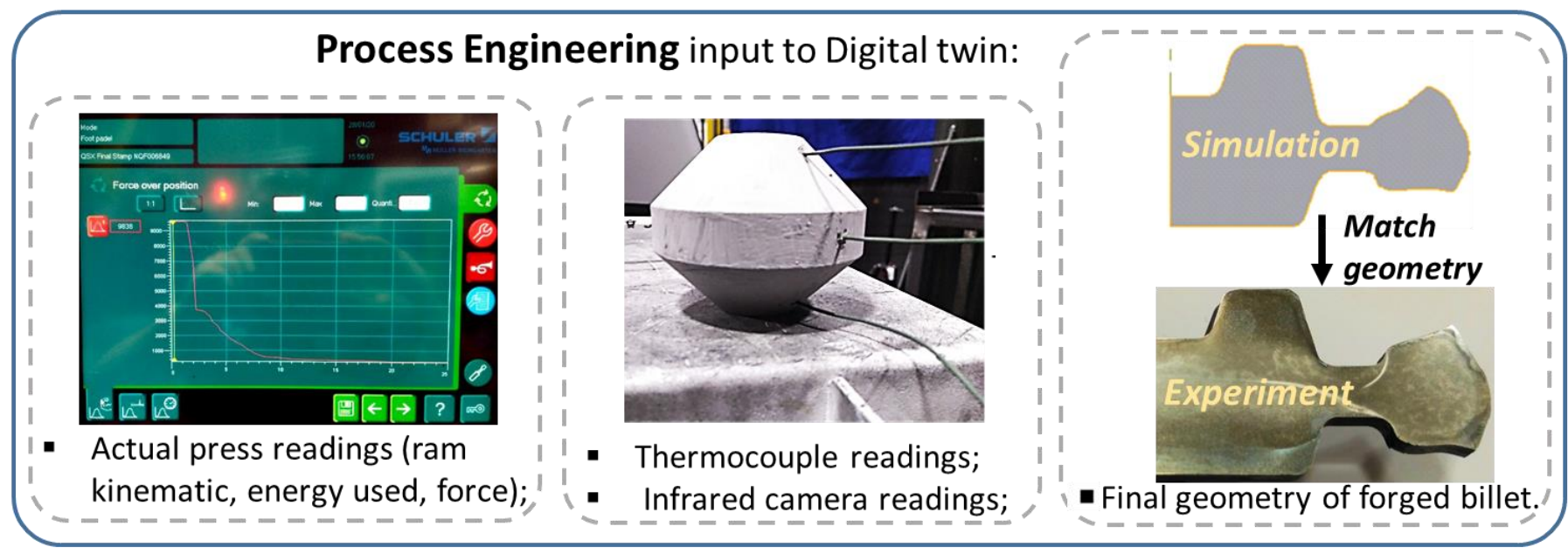

\section{Mechanics of Solids input to Digital twin:}

- Model of the material $\sigma=f(\varepsilon, \dot{\varepsilon}, T)$;

- Flow stress data extra-\&interpolation.

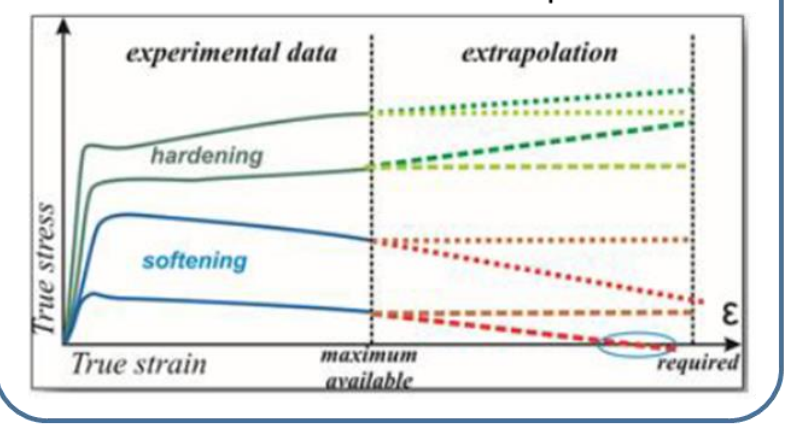

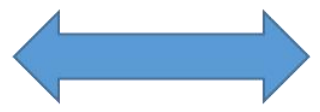

Calibration by:

- Energy;

- Force;

- Flow lines.
Calibration by:

- Temperature;

- Final shape.

\section{Digital Twin}

of the process

(adjusting initial FE model to actual forging)

Figure. 5. Schematic of the steps involved in construction of a digital twin.

Typical FE models are created prior to the actual forging to assess the effectiveness of the process conditions and predict forging requirements such as load and energy. There are many simplifying assumptions regarding the boundary conditions and material flow stress, but these are overlooked as long as the expected results fall within a reasonable margin of error and can be used for assessment. Digital twins, in contrast, are constructed after the forging process typically using the same FE package, but made to imitate the actual process as closely as possible. All the data collected from the actual trials are fed into the FE model and the level of confidence in the model is improved, similar to an inverse problem. To overcome the uncertainties in replicating the tool kinematics, friction conditions, heat exchange and material flow, the following steps were adopted:

1. To replicate the actual kinematics of the process, real-time velocity-vs-position data recorded in the press was fed into the model. 
2. Actual time of each operation (heating in furnace, transfer to the dies, resting time on die before forging, forging operation, resting after forging, transfer to quench tank and quenching) were obtained using video-recordings and input into the FE model.

3. Temperature data from carefully placed thermocouples were used to reconstruct the heat exchange in billet with air and the dies. Three thermocouples with their tips aligned with the central axis were placed along top, middle and bottom as shown in Fig. 6 below. The temperature profiles obtained for the 3 points were used for inverse adjustment of heat transfer coefficients in the digital twins.

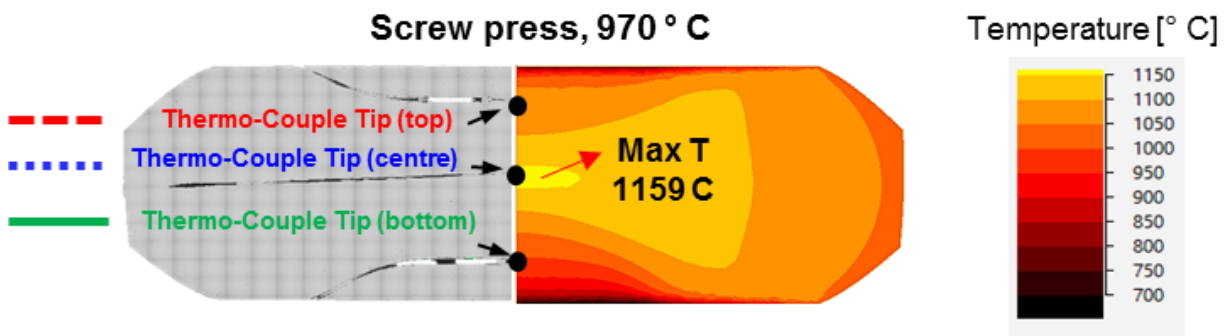

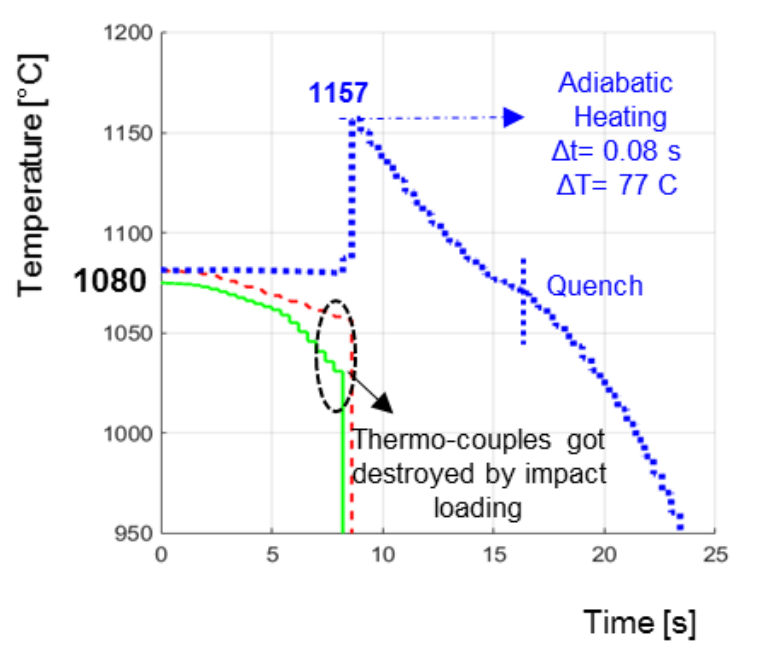

(b)

(a)

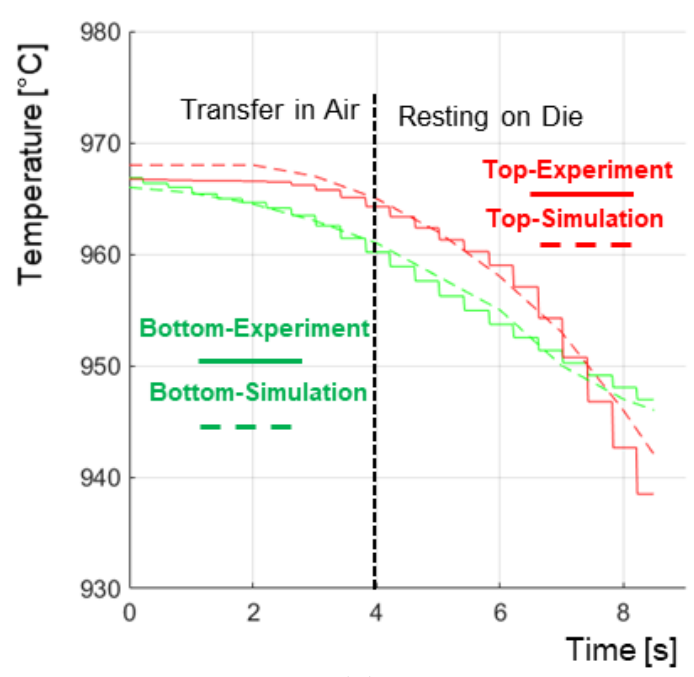

(c)

Figure 6. Thermocouple readings from screw press forging trials: (a) DTC geometry with embedded thermocouples; (b) actual thermocouple reading (with the top blue line representing the temperature recorded at the centre of the cross-section); (c) validating the temperature readings in the digital twin using tracking points placed at the exact locations

4. Friction parameters were adjusted by matching the actual geometry and flow lines observed in macrographs from experiments to those from simulations for both partially and fully forged billets, as shown in Fig. 7.

5. The initial series of iterations were simulated using ideal plastic flow stress data obtained from the literature $[14,16]$. These in turn were obtained from uniaxial compression tests carried out to a strain of 0.7 at a number of strain-rates and temperatures. After fine-tuning the friction and temperature settings in the model, the flow stress data was extrapolated as the last step. The metal flow from macrographs and force recordings from the press were used as a reference for this. 


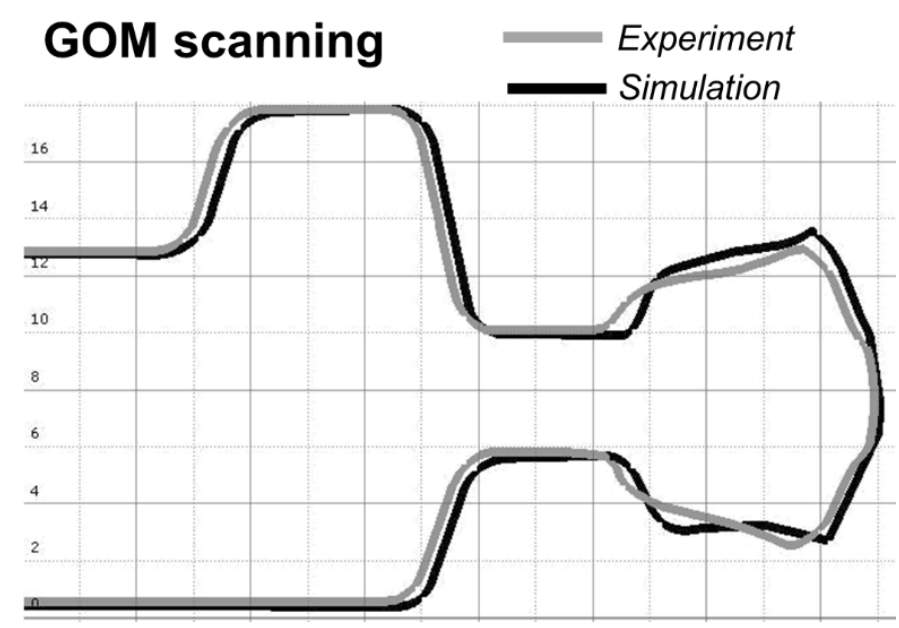

(a)

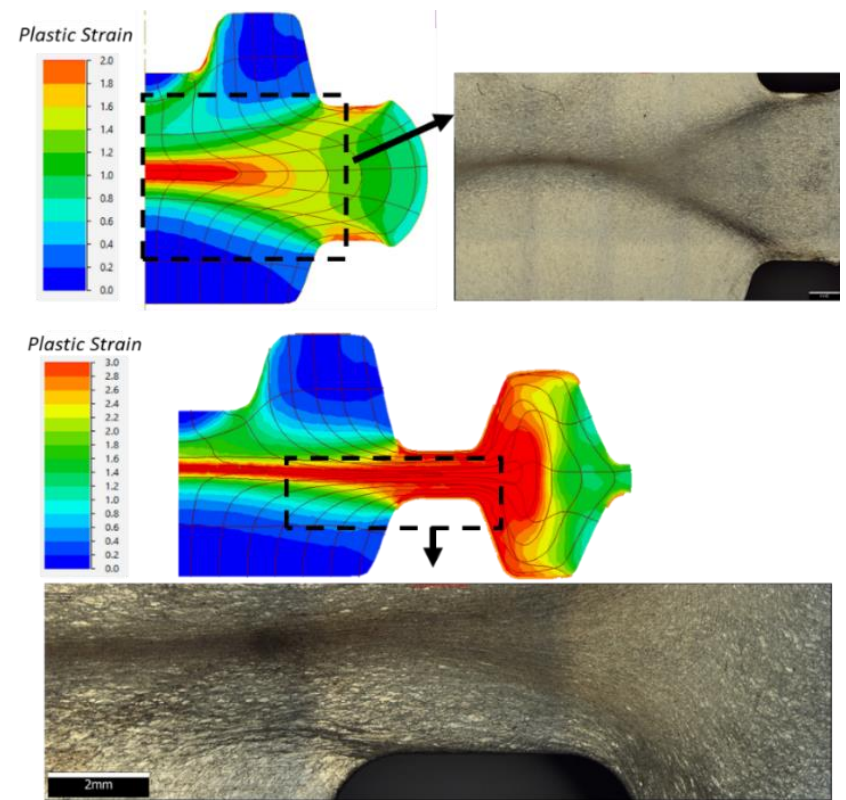

(b)

Figure 7. Matching final geometry shape in simulation with actual geometry of forged part: (a) matching geometry using obtained a 3D blue-light based metrology scanning system (by GOM.com) by adjusting friction parameters; (b) adjusting metal flow in simulation using macrographs of shear bands from an Alicona scanner

\section{3) Extracting trajectories of loading history}

The third important part of the methodology involves output of robust data that can be used for calibration, validation and improvement of material and microstructural models. This is suggested in the form of trajectories of loading (i.e. thermo-mechanical history) for key points of interest within the forged disk obtained from the digital twin of the process. For instance, regions of unstable flow and unusual microstructural evolution can be identified within the forged component. Tracking points (sensors) can be placed in these regions in the FE package and tracked back to the start of the process. Thus, the history of the strain, strain rate and temperature evolution in these points can be extracted through the process and plotted in the space of strain-vs-temperature-vs-strain-rate. Figure 2 showed an example of the trajectories of reference points plotted for the disk geometry forged in this study. This is also contrasted with the trajectory of points expected from uniaxial compression tests of cylindrical samples. Using this approach, the material models can be validated and calibrated based on more realistic values of strain, strain-rate and temperature and their history. This is especially useful in the case of high-strain-rate forging, as it involves adiabatic heating, which causes difficulties in processing of results from lab-scale high-strain-rate tests. This also can provide additional information on the material science behind many microstructural phenomena happening in FIFP. For instance, time can be substituted for one of the axes, and the effect of the process time can be correlated with the microstructural transformations observed.

\section{Results and Discussion}

The above-mentioned methodology of creation of digital twins and reconstruction of thermo-mechanical histories in different points in the forged part opens up new horizons in studying FIFP of nickel-based superalloys. Some successful case studies where this approach has provided new and interesting insights into closed-die screw press forging of a disk geometry using IN718 are described here. As stated, this approach can easily be translated to other forging conditions and materials to open further possibilities into the study of FIFP.

Example\#1: Identification of thermo-mechanical history that leads to the onset of specific material flow or microstructural phenomena

Thermo-mechanical history from digital twins can be a useful tool to identify and understand the conditions which result in specific metal flow or microstructural transformation behaviour. For instance, in cases where 
abnormal grain growth, the beginning of recrystallisation (RX) or dissolution of secondary phases occur in specific zones in the forged part, multiple tracking points can be placed in these zones in the digital twin and tracked back to obtain the complete history of loading through the process. If these behaviours repeat under different processing conditions, the trajectories can be extracted for all of them and compared. If peculiarities are observed in the trajectories, this can be used to formulate a criterion in models to predict the onset of the phenomena. This is not easily possible in homogenous uniaxial tests, as it is almost impossible to stop the process exactly at the onset of a particular phenomenon, especially in a high-strain-rate process.

Figure 8 below shows an approach adopted to study the onset of RX in forged disks of IN718. Figure 8(b) shows the boundary (isoline) for onset of RX in the forged disk. As can be seen, different points along the isoline have quite different thermo-mechanical histories. Since the forging was essentially non-isothermal and non-monotonic, it is not unusual that different trajectories lead to the same phenomena in the forging. Further studies can be made to explore all possible combinations of strain, strain-rate and temperature history that lead to the onset of RX, and these data can be used for calibration of an industry-specific RX model [6]. This way, data from real-time industrial forging processes can be input to validate, calibrate or even create industryspecific models, thus avoiding the complicated task of adjusting lab-based models to industry needs.

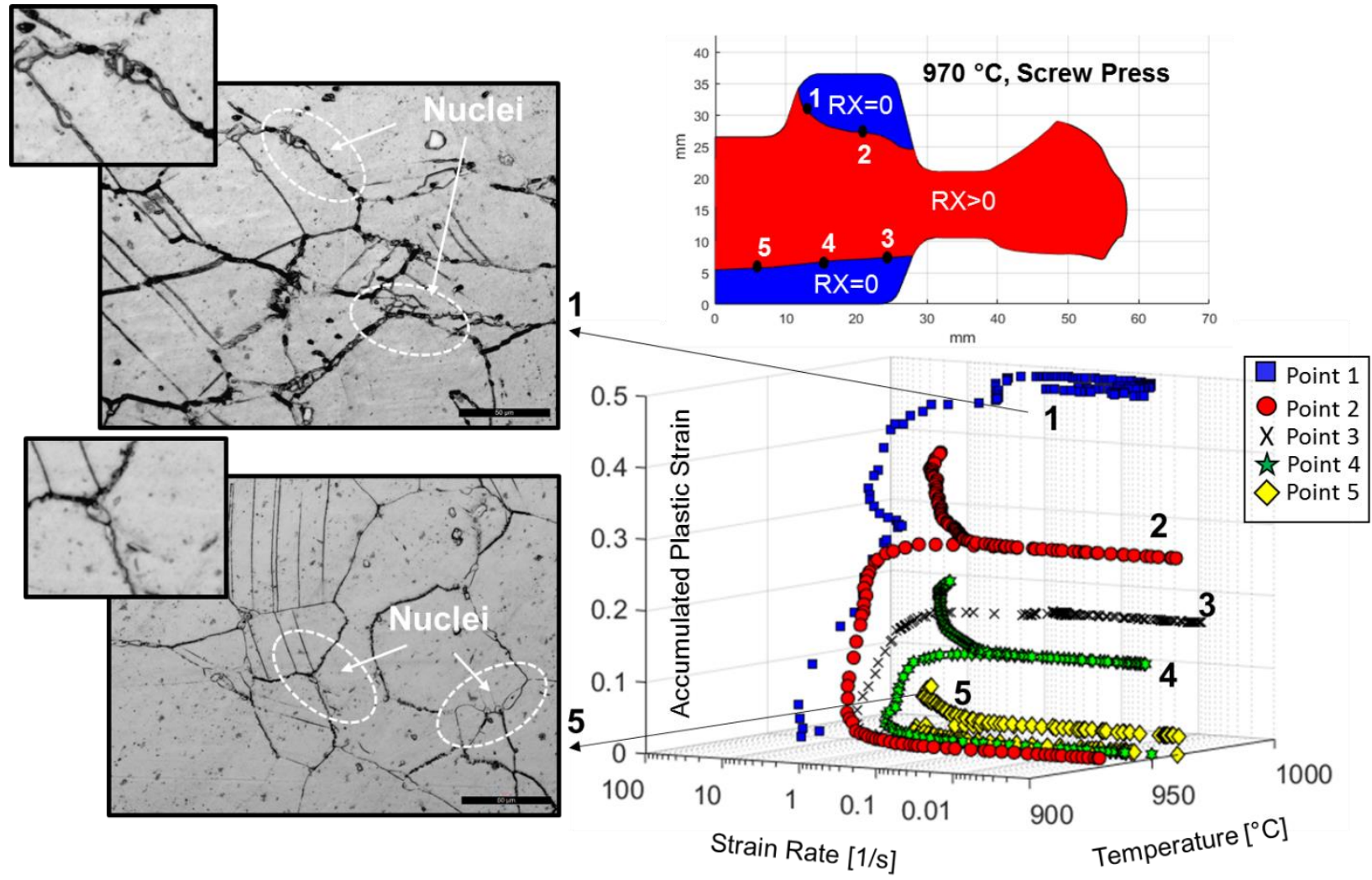

(a)

(b)

Figure 8. Onset of recrystallisation in forged disc geometry: (a) SEM images of reference points in disk's cross-section illustrating onset of RX; (b) location of the reference points (top-right image) and plot of thermomechanical histories for the points where RX initiated.

Example\#2: Analysis of the influence of selected process parameter on the thermo-mechanical history and final microstructure.

Another useful application of this methodology is the possibility of comparing the trajectories of analogous points in similar geometries forged with different process parameters. For instance, it is possible to compare the trajectories of points in disks forged at two different temperatures or strain rates, especially high strainrates, maintaining all other process parameters the same. This is a unique feature of the digital twin, as it is almost impossible to maintain the temperature or strain-rate constant under industrial conditions. Complicated as-forged geometries, the tendency to see variance in press outputs and numerous other factors render the prospect of reproducing such conditions using lab-based experimental trials unlikely. For any meaningful comparison, it is required to bring the processes compared to a common baseline. In this case, we can aim to 
perform experiments as close to the desired conditions as possible, develop a digital twin of the process and then correct for any deviations caused by realistic conditions.

Figure 9 below shows the results from our studies to understand the effect of strain-rate on the microstructural evolution during industrial forging of IN718. As touched upon briefly before, the microstructural transformation at high strain rates and strains differs from the well-studied deformation at low strains and velocities of loading. Fig. 9 shows the microstructures obtained in the middle of a part on forging with a hydraulic press (slow strain-rate) and screw press (high strain-rate). The figure shows the strain, strain-rate, and temperature processing window and as will be noted these forgings go well beyond the strain range of uniaxial tests, and as such, the microstructure evolution cannot be modelled or predicted using the classical approach. The trajectory for a point in the middle of the part forged on the screw press differs completely from that observed for a hydraulic press. Consequently, the RX\% vol. and resulting microstructure differ drastically. Further studies can be carried out using this approach to formulate criteria and model the microstructural transformation as a function of the process parameters.

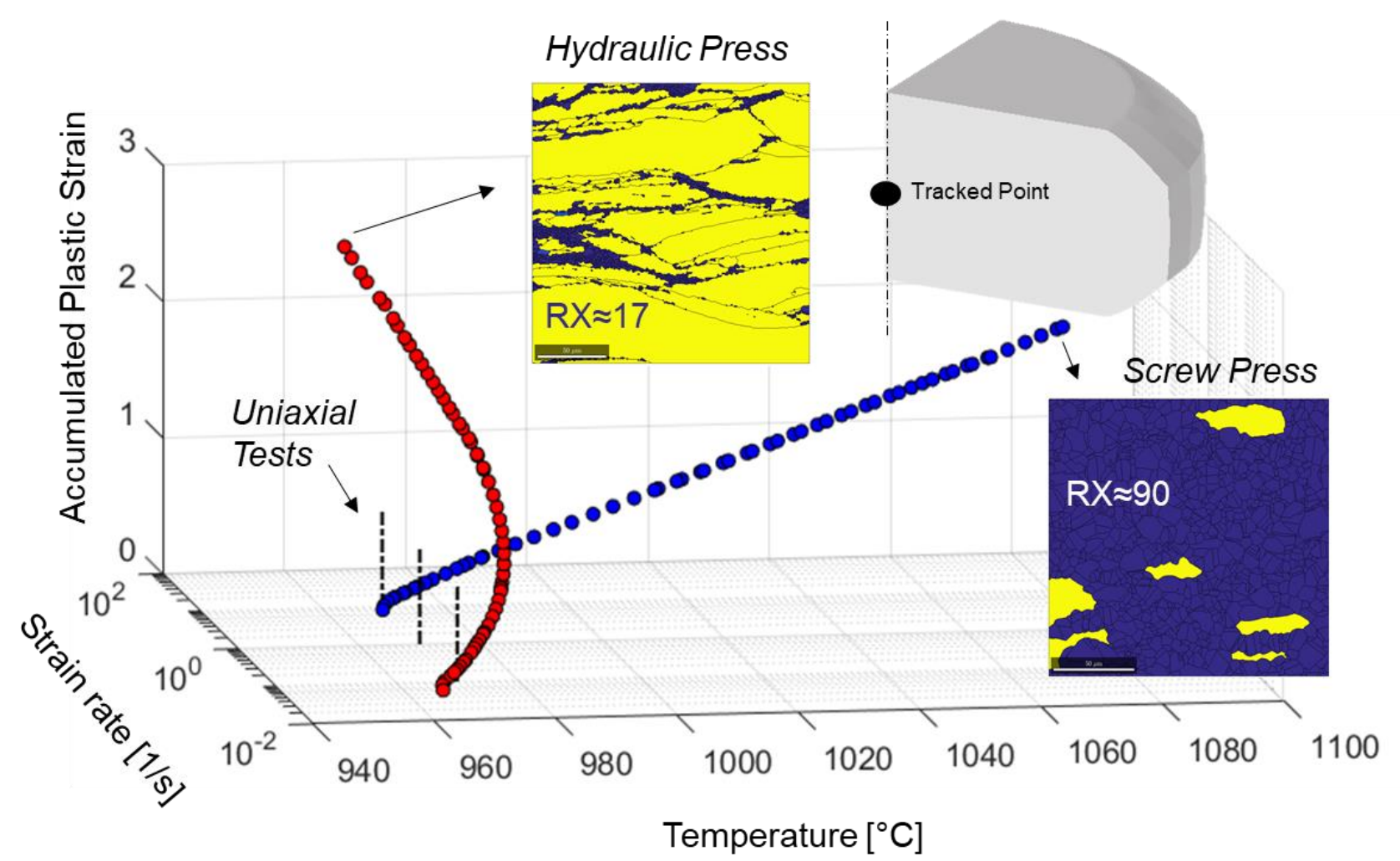

Figure 9. Studying influence of strain rate parameter on the microstructure evolution of IN718 and trajectory of loading: thermo-mechanical history and final microstructural state in central point of the DTC cross-section forged on hydraulic (red dotted trajectory) and screw press (blue dotted trajectory)

Example\#3: Study of microstructure at various stages of the forging operation (reconstruction of microstructure evolution during one blow).

The evolution of microstructure in FIFP is another area of study where the use of digital twins and loading history can be hugely helpful. They can provide a wealth of information to improve our material science understanding of many microstructural phenomena happening in FIFP. As emphasised, while microstructure images provide instantaneous information about the microstructure changes at a particular stage of forging, they do not provide insights into the nature of evolution and how it is linked to the history of loading. The suggested methodology can link the microstructure images to macro- and meso-level parameters used and observed in actual industrial forging and thus provide a tool to answer questions related to their evolution.

Figure 10 below shows the results from a study of microstructure evolution at various stages of forging of IN718 disks using a screw press. Two sets of forgings, one stopped midway using a spacer plate, and another completed, were carried out using the same set of parameters (temperature $970^{\circ} \mathrm{C}$ and energy $120 \mathrm{~kJ}$ ). Two points closely placed next to each other were identified in the zone of interest in the digital twin of the fully 
forged component, namely, Point\#7 and Point\#8. These points were tracked back to extract the full history of loading. Furthermore, the positions of these points were tracked back to the half-forged condition. Thus, microstructure images were obtained for the half-forged conditions at these exact points for comparison using the position information. It can be observed from Fig. 10(b) that the evolution differs notably for two closely aligned points. It can be observed that at Point $\#$, the microstructure is completely devoid of recrystallisation under the half-forged condition, while there is partial recrystallisation under the fully forged condition. In contrast, Point\#8 exhibits recrystallisation at both stages. When studied together with the loading history, it can be observed that Point\#7 stayed in the dead zone at mid-forging and it started accumulating deformation energy much later than did Point\#8. This information might go unnoticed when looking at just the microstructure images and can be very useful for understanding the images and the microstructure evolution in general. This valuable information can then be employed in improvement and development of microstructural evolution models.

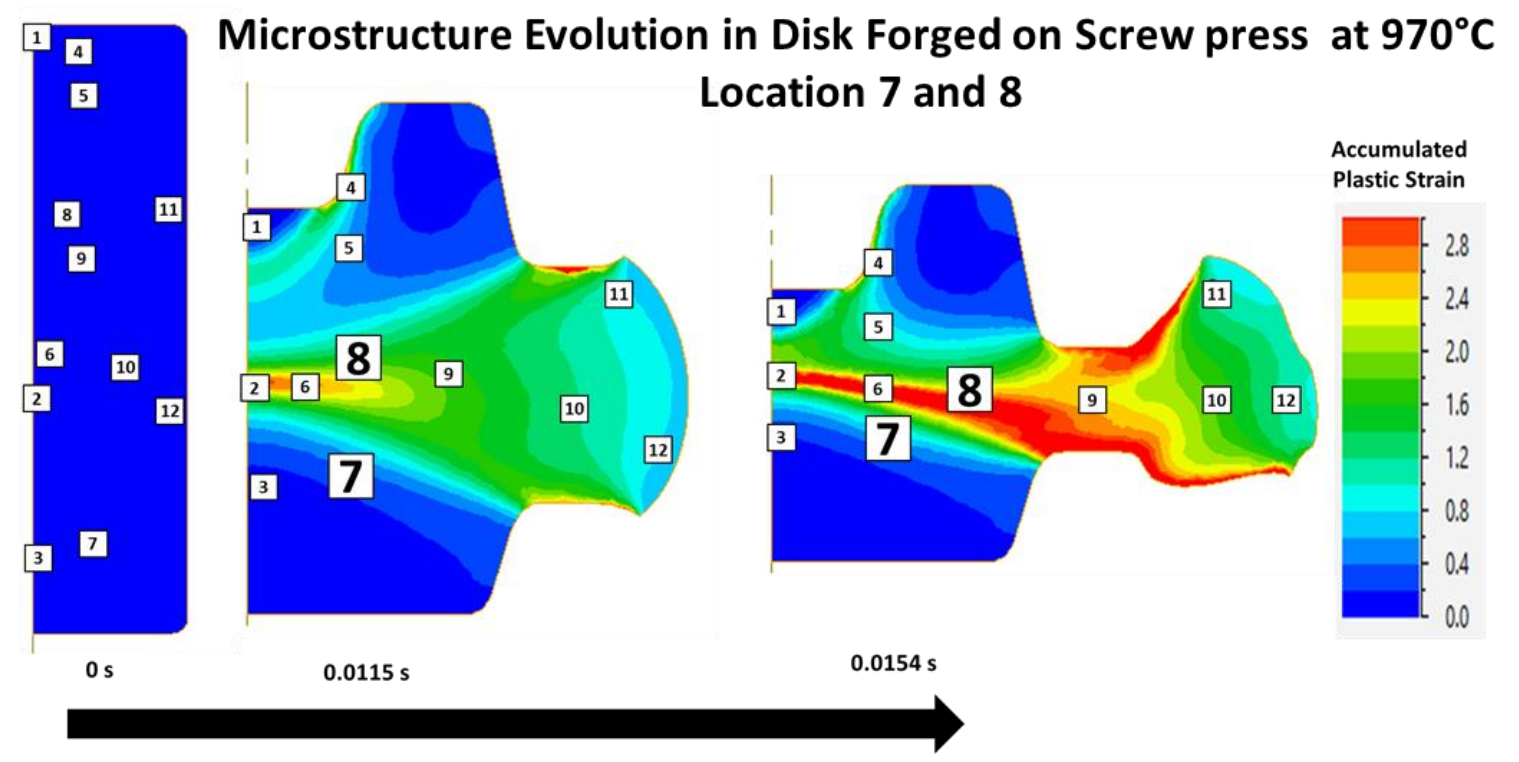

(a)
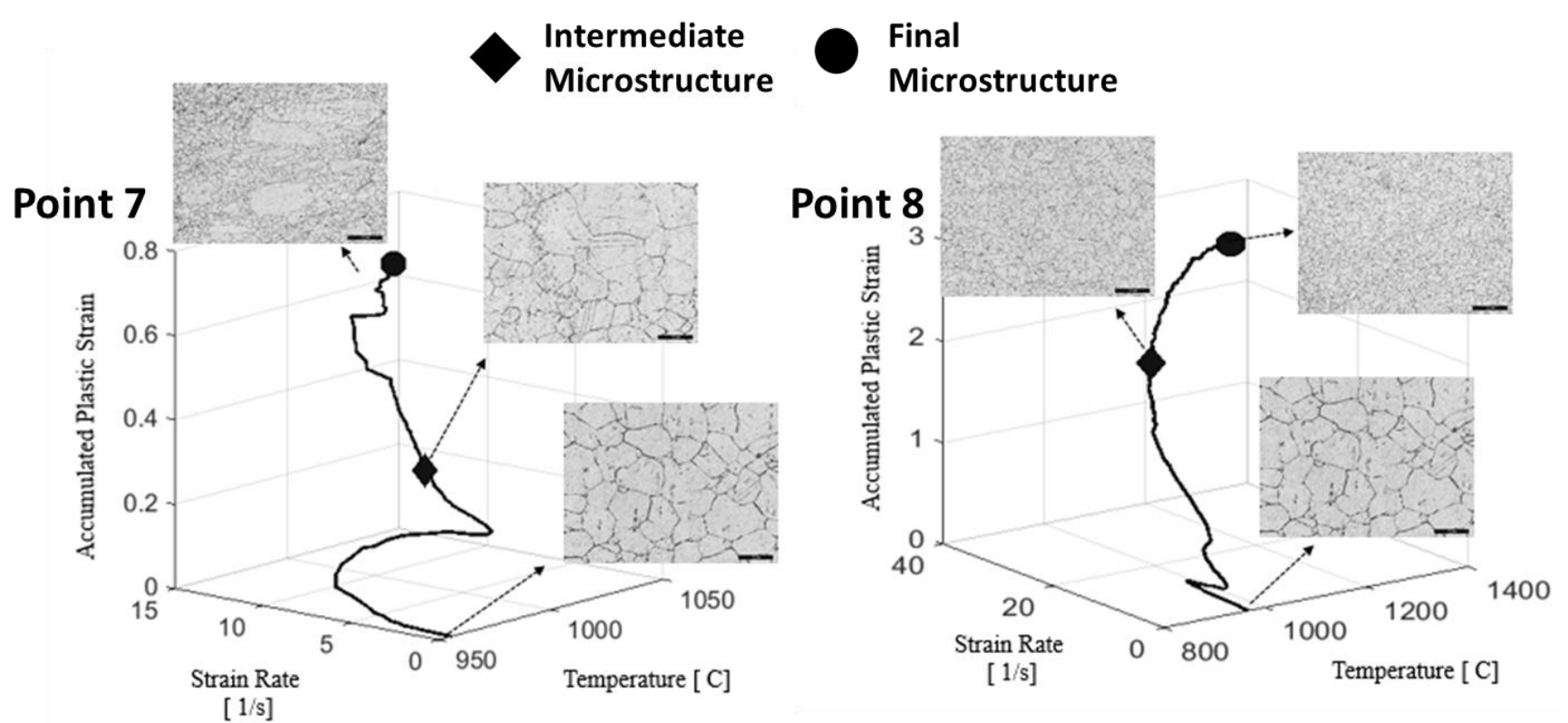

(b)

Figure 10. Studying microstructure at various stages of the forging operation: (a) back-tracking reference points with digital twin of the process; (b) example of microstructure evolution in points\#7 and \#8 superimposed with their thermo-mechanical history. 


\section{Conclusions}

This work addresses a main problem of complex industrial forgings - insufficiency of material data obtained from uniaxial lab tests for adequate modelling of the process and microstructural transformation. Herein, a hybrid experimental-computational methodology is proposed for digitising, correlating and processing mechanical and microstructural data from significantly heterogeneous industrial forgings. Although this methodology does not resolve the problem of mixing and overlapping of different microstructural mechanisms acting through the process, it can be a powerful tool for Design of Experiments and analysis of complex processes. It also provides a good platform for calibration and validation of constitutive and microstructural models as well as material science hypotheses.

\section{References}

[1] Sims CT (1984) A History of Superalloy Metallurgy for Superalloy Metallurgists. Superalloys 399419. doi:10.7449/1984/Superalloys_1984_399_419

[2] Cao WD, Kennedy RL (2004) Role of chemistry in 718 Type Alloys-Allvac $\left(718\right.$ Plus ${ }^{\mathrm{TM}}$ alloy development. Superalloys 3:91-99. doi: 10.7449/2004/Superalloys_2004_91_99

[3] Xie XS, Dong JX, Zhang MC (2009) Research and Development of Inconel 718 Type Superalloy. Mater. Sci. Forum. 539-543:262-269. doi:10.4028/www.scientific.net/msf.539-543.262

[4] Altan, T, Ngaile,G, Shen, G (2005) Cold and Hot Forging. ASM International, Ohio

[5] Jaeger JDe, Solas D, Baudin T, Fandeur O, Rey C (2012) Inconel 718 single and multipass modelling of hot forging. Superalloys 663-672. doi: 10.1002/9781118516430.ch74

[6] Bylya O, Reshetov A, Stefani N, Rosochowska M, Blackwell P (2017) Applicability of JMAK-type model for predicting microstructural evolution in nickel-based superalloys. Procedia Eng. 207:11051110. doi:10.1016/j.proeng.2017.10.1067

[7] Stefani N, Bylya O, Reshetov A, Blackwell P (2017) On the applicability of JMAK-type models in predicting IN718 microstructural evolution. Comput. Methods Mater. Sci. 17:59-68

[8] Tang B, Xiang L, Cheng L, Liu D, Kou H, Li J (2018) The Formation and Evolution of Shear Bands in Plane. Metals (Basel). 8:1-11. doi:10.3390/met8020141

[9] Bylya OI, Sarangi MK, Nayak A, Vasin RA, Blackwell PL (2015) Simulation of the material softening during hot metal forming. Arch. Metall. Mater. 60:1887-1893. doi:10.1515/amm-20150322

[10] Nicolaÿ A, Fiorucci G, Franchet JM, Cormier J, Bozzolo N (2019) Influence of strain rate on subsolvus dynamic and post-dynamic recrystallization kinetics of Inconel 718. Acta Mater. 174:406417. doi:10.1016/j.actamat.2019.05.061

[11] Thomas JP, Bauchet E, Dumont C, Montheillet F (2004) EBSD investigation and modeling of the microstructural evolutions of superalloy 718 during hot deformation. Superalloys 959-968. doi:10.7449/2004/superalloys_2004_959_968

[12] Dumont C, Georges E (2011) Microstructure prediction during incremental processes for hot forming of 718 alloy. Adv. Mater. Res. 278:186-191. doi:10.4028/www.scientific.net/AMR.278.186

[13] Srinivasan N, Prasad YVRK (1994) Microstructural Control in Hot Working of IN-718 Superalloy Using Processing Map. Metall. Mater. Trans. A. 25:2275-2284. doi:10.1007/BF02652327

[14] Sui F, Xu L, Chen L, Liu X (2011) Processing map for hot working of Inconel 718 alloy. J. Mater. Process. Tech. 211:433-440. doi:10.1016/j.jmatprotec.2010.10.015

[15] Bylya O, Gomez-Gallegos A, Stefani N, Blackwell P (2017) Al-Li alloys - the analysis of material behaviour during industrial hot forging. Procedia Eng. doi: 10.1016/j.proeng.2017.10.729

[16] Prasad YVRK, Rao KP, Sasidhara S (2015) Hot Working Guide. A Compendium of Processing Maps. ASM International, Ohio 